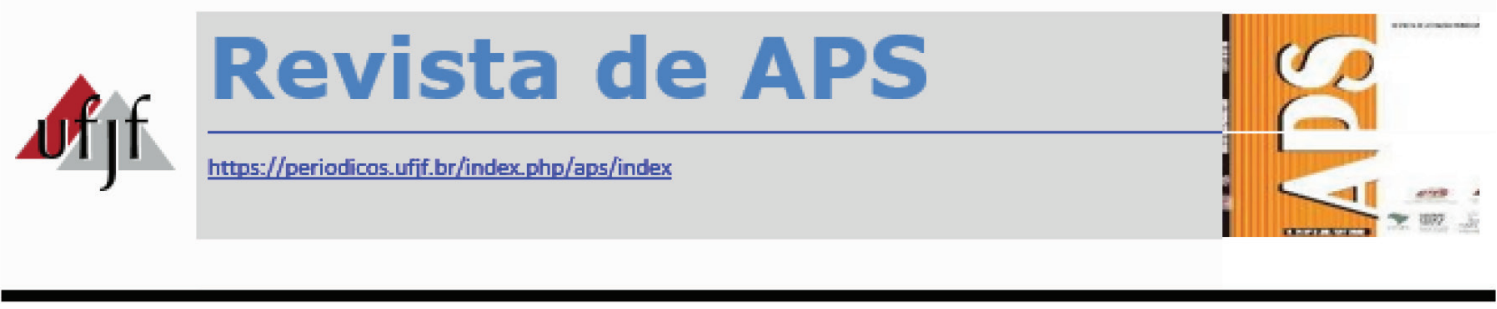

\title{
A Assistência Farmacêutica no município de Juiz de Fora em Minas Gerais: uma visão a partir dos Planos Municipais de Saúde
}

\author{
The Pharmaceutical Assistance in the municipality of Juiz de Fora in \\ Minas Gerais: a look from the municipal health insurance plans
}

Maria Helena Braga ${ }^{1}$, Patrícia Aparecida Baumgratz de Paula ${ }^{2}$, Terezinha Noemides Pires Alves ${ }^{3}$

\section{RESUMO}

O presente estudo teve como objetivo discutir a Assistência Farmacêutica (AF) no município de Juiz de Fora, inserida nos Planos Municipais de Saúde (PMS), no período de 1988 a 2017. A pesquisa parte de revisão bibliográfica e avança pelo campo da investigação documental. Para contextualização do cenário foi utilizado o resgate histórico da AF no Brasil e no estado de Minas Gerais em recorte a partir dos anos 1980 tendo por base documentos, leis e portarias. A fonte principal da pesquisa inclui a busca, a leitura e a categorização dos Planos Municipais de Saúde de Juiz de Fora/MG, nos seguintes períodos: 1988; 1997; 2002/2005; 2006/2009; 2010/2013; e 2014/2017. Para a avaliação da assistência farmacêutica apoiou-se nos indicadores da Organização Pan-Americana da Saúde, de 2005: acesso, qualidade e uso racional de medicamentos. $O$ estudo demonstrou que os indicadores acesso aos medicamentos e uso racional dos medicamentos foram, de certo modo, contemplados nesses planos. Todavia, o indicador qualidade dos medicamentos ainda se encontra incipiente. Foi possível concluir que a AF avançou no período com a introdução da Assistência Farmacêutica no organograma da Secretaria Municipal de Saúde, criação do Departamento de Assistência Farmacêutica (DAF), implantação da Comissão de Farmácia e Terapêutica (CFT), presença do farmacêutico como gestor da $A F$, aumento do número desses profissionais no quadro funcional do município e criação de uma equipe para acompanhar os processos de aquisição de medicamentos. No entanto, ainda são identificadas fragilidades em relação ao

\footnotetext{
${ }_{1}^{1}$ Professora Aposentada da Universidade Federal de Juiz de Fora, Doutora em Saúde Coletiva.

${ }^{2}$ Professora Adjunta da Universidade Federal de Juiz de Fora - Campus Governador Valadares, Doutora em Saúde Coletiva. E-mail: patricia.paula@ufjf.edu.br

${ }^{3}$ Professora Aposentada da Universidade Federal de Juiz de Fora, Doutora em Saúde Coletiva.
} 
sistema de informação sobre os medicamentos e gestão da AF, ao acesso racional aos medicamentos, falta e desperdício de medicamentos, à não implantação do NASF, bem como à discussão e materialização das farmácias distritais, a exemplo de outros municípios mineiros, polos das macrorregiões de saúde.

PALAVRAS-CHAVE: Assistência Farmacêutica. Controle Social. Uso de Medicamentos.

\section{ABSTRACT}

The present study had as an objective to discuss the Pharmaceutical Assistance (PA) in the city of Juiz de Fora, inserted in the Municipal Health Plans (MHP), in the period from 1988 to 2017. The research sets off from the bibliographic review and advances through the field of the documental investigation. For the scenery contextualization, the historical rescue of the PA in Brazil and in the state of Minas Gerais was used, from the 1980s having, as base documents, laws and directives. The main source of the research includes the researching, reading and categorizing of the Municipal Health Plans of Juiz de Fora/MG, in the following periods: 1988; $1997 ; 2002 / 2005 ; 2006 / 2009 ; 2010 / 2013$ and 2014/2017. For the evaluation, the pharmaceutical assistance based itself in the Pan-American Health Organization, from 2005: access, quality and the rational use of medication. The study shows that the indicators access to medication and rational use of medication were, in a certain way, contemplated in these plans. Even so, the quality indicator of the medication remains incipient. It was possible to conclude that the PA advanced in this period with the introduction of the Pharmaceutical Assistance in the Municipal Secretariat's organizational chart, the creation of the Pharmaceutical Assistance Department (PAD), implementation of the Therapeutic Pharmacy Commission (TPC), presence of the pharmaceutical professional as manager of the PA, increase in the number of these professionals in the city's health service and creation of a team to follow the medicament acquisition procedures. Although, there can still be identified frailties in relation to the information concerning the medication and management of the PA; the rational access to medication, lack and waste of medication; non implantation of the FHSN, as well as, the discussions about and implantation of the districtal pharmacies, following the example of other cities in the state, poles of the health macroregions..

KEYWORDS: Pharmaceutical Services. Social Control. Drug Utilization

\section{INTRODUÇÃO}

O presente trabalho procurou discutir a inserção e o desenvolvimento da Assistência Farmacêutica (AF) no município de Juiz de Fora - MG, tendo como base os Planos Municipais de Saúde (PMS) e, como pano de fundo, um breve resgate histórico da AF no Brasil e no estado de Minas Gerais.

Observou-se, na leitura dos referidos documentos, a presença de avanços em alguns marcos regulatórios na AF, principalmente no final da década de 1990 e início dos anos 2000, com a formulação do Plano Municipal de Assistência Farmacêutica (1999) e a inserção da AF nas discussões do Conselho Municipal de Saúde, além da criação do Departamento de Assistência Farmacêutica (DAF) do município, pelo Decreto 9.747/09¹. 
Apesar disso, a AF continua necessitando de maior atuação dos gestores, pois ainda existem diversos problemas identificados nas análises realizadas principalmente pela constante falta e/ou insuficiências de medicamentos e a ausência de farmacêuticos para efetiva AF nas Unidades Básicas de Saúde (UBS).

A criação, pela Constituição Federal em 1988, do Sistema Único de Saúde (SUS) colocou a saúde como direito social na agenda das políticas públicas de saúde no Brasil. Apesar de o período pós-Constituição ter sido marcado por governos baseados no ideário neoliberal, as Leis Orgânicas da Saúde conferiram materialidade ao SUS nos anos de 1990.

A Lei $\mathrm{n}$ - 8.080/90 dispôs sobre a organização político-administrativa definindo a gestão compartilhada do sistema de saúde distribuída nas três esferas de governo, assumindo as responsabilidades de promoção, prevenção e recuperação da saúde, bem como o financiamento destas ações ${ }^{2}$. No mesmo ano, a Lei no 8.142, em seu artigo 10, tratou da participação da comunidade na gestão do SUS definindo as Conferências e Conselhos de Saúde como instâncias formais do controle social, e seu Art. 4으 define a necessidade da elaboração dos Planos de Saúde (PMS) e das suas prerrogativas ${ }^{3}$.

Ao dar continuidade ao processo de abertura democrática, iniciado na década de 1990, ampliaram-se as discussões sobre a garantia do acesso aos medicamentos como direito de cidadania que culminaram com a instituição da Política Nacional de Medicamentos (PNM) pela edição da Portaria GM/MS № 3.916/984. Tal portaria instituiu, no Ministério da Saúde (MS), o Departamento de Assistência Farmacêutica (DAF) fortalecendo as estratégias para a garantia ao acesso integral, equânime e universal aos medicamentos no SUS, incluindo os insumos estratégicos na área de saúde. Em continuidade à implementação das diretrizes e prioridades da PNM, o MS publicou a Portaria № 176/99 que "estabelece critérios e requisitos para a qualificação dos municípios e estados ao Incentivo à Assistência Farmacêutica Básica e define valores a serem transferidos" ${ }^{\prime \prime}$.

Cabe ressaltar que nesse período foi extinta a Central de Medicamentos (CEME) que, entre outras prerrogativas, possuía as funções de aquisição e distribuição de medicamentos, assumindo sua regulação. Segundo Kornis, Braga e Zaire ${ }^{6}$, a forma fragmentada como a CEME desenvolvia suas atividades, a desarticulação do setor público e a hegemonia das empresas farmacêuticas transnacionais no mercado farmacêutico brasileiro foram determinantes para a sua desativação, ocorrida em 1997.

Articulada ao controle social e com uma lacuna em torno de 10 anos, a AF vai sendo inserida na gestão da saúde de forma sistemática. Em 2003, foi realizada a Conferência Nacional de Medicamentos e Assistência Farmacêutica tendo, como objetivo principal, analisar a situação do serviço de Assistência Farmacêutica no SUS, sob o tema central "Acesso, Qualidade e Humanização da Assistência Farmacêutica com Controle Social". Importante consequência desta conferência foi a aprovação, pela Resolução no. 338/04 
do Conselho Nacional de Saúde, da Política Nacional de Assistência Farmacêutica (PNAF) trazendo definições sobre assistência e atenção farmacêuticas e enfocando a política de medicamentos ${ }^{7}$.

A Portaria GM no 399/06 instituiu o Pacto pela Saúde estabelecendo blocos de financiamento para o custeio do SUS, sendo um deles o bloco da assistência farmacêutica, com atividades voltadas para promover a estrutura da AF e garantir o acesso da população aos medicamentos e seu uso racional, em conjunto com as demais esferas de governo ${ }^{8}$. Do mesmo modo, a Portaria GM no 204/07 regulou a forma de financiamento e transferência de recursos para as ações e serviços de saúde. Nesta, o bloco da AF contemplava três componentes: i) básico da assistência farmacêutica, ii) estratégico da assistência farmacêutica, e iii) medicamentos de dispensação excepcional. Cada um dos componentes receberia recursos em conta específica, os quais deveriam ser aplicados exclusivamente nas ações definidas para cada componente. O componente básico visava à aquisição de medicamentos e insumos relacionados à atenção básica em saúde, como também para programas específicos que faziam parte da atenção básica. Possuía uma parte fixa, valor transferido da União para os Estados e Municípios, a partir de pactuações na Comissão Intergestora Bipartite (CIB), e outra parte variável. Os Estados e Municípios deveriam dar uma contrapartida para a parte fixa. Já a parte variável estava relacionada aos diversos programas, como: Hipertensão e Diabetes, Asma e Rinite, Saúde Mental, Saúde da Mulher, Alimentação e Nutrição e Combate ao Tabagismo. O componente estratégico atendia a programas de saúde estratégicos como controle de endemias, antirretrovirais do programa IST/AIDS, sangue e hemoderivados e imunobiológicos. Já o componente medicamentos de dispensação excepcional relacionava-se ao grupo de medicamentos dispensados segundo critérios estabelecidos em portarias, sendo de responsabilidade do MS e dos Estados ${ }^{9}$.

Vale ressaltar as ações estratégicas promovidas pelo DAF, como a criação do Comitê Nacional para a promoção do Uso Racional de Medicamentos, em julho de 2007, buscando identificar e propor estratégias de articulação, de monitoramento e avaliação da promoção do uso racional de medicamentos.

Nessa perspectiva, a evolução das políticas relacionadas aos medicamentos no âmbito do SUS, na primeira década de 2000, deu centralidade à assistência farmacêutica com foco no cuidado farmacêutico, com o envolvimento das esferas federal, estadual e municipal. Como afirmam Correr, Otuki e Soler ${ }^{10}$, a AF constitui um dos sistemas de apoio das redes de atenção à saúde, com ênfase na garantia do acesso com uso racional de medicamentos, envolvendo a gestão técnica da assistência farmacêutica e a gestão clínica do medicamento, que se configura como uma atividade assistencial fundamentada no processo de cuidado. Todavia, a extensão territorial e a questão federativa do Brasil, marcada principalmente pela presença de muitos municípios com menos de 10.000 habitantes, dificultam a gestão da assistência farmacêutica. 
No início da segunda década de 2000, a agenda política para os medicamentos avançou na área da ciência e tecnologia. A Lei no 12.401/11, que dispôs sobre a assistência terapêutica, a incorporação de tecnologia em saúde no âmbito do SUS e sobre a Comissão Nacional de Incorporação de Tecnologias (CONITEC), considerou a dispensação de medicamentos como parte da assistência terapêutica integral ${ }^{11}$. Também nesse ano foi editado o Decreto no 7.508, que tratou da organização do SUS, do planejamento da saúde, da assistência à saúde e da articulação entre os entes federais ${ }^{12}$.

Paralelamente às estratégias desenvolvidas pelo núcleo central para a área de medicamentos, na primeira década de 2000, destacam-se as seguintes experiências nas esferas estaduais: i) em 2004, iniciativa do estado de São Paulo ao criar o Programa Dose Certa, com o objetivo de aperfeiçoar o acesso da população aos medicamentos no âmbito da atenção básica, por meio do fornecimento de medicamentos, que são produzidos, em parte, pela Fundação para o Remédio Popular (FURP) ${ }^{13}$; ii) criação do Programa Farmácia Cidadã, no Espírito Santo em 2008, visando à ampliação do acesso aos medicamentos e ao aprimoramento da qualidade dos serviços ofertados aos usuários de medicamentos ${ }^{14}$; e iii) em 2007, Minas Gerais implementou o Programa Rede Farmácia de Minas (RFMG) ${ }^{15}$.

\section{Marcos da assistência farmacêutica no estado de Minas Gerais}

Os primeiros marcos regulatórios da AF são identificados no estado de Minas Gerais, a partir da década de 1980, com as ações do Programa Farmácia Básica, pari passu com as atividades voltadas para a atenção básica, um dos focos da gestão da Central de Medicamentos (CEME 1971-1997).

Em maio de 1997, Minas Gerais implantou o Projeto Farmácia Essencial (PFE), enquanto política de medicamentos para o nível primário de saúde que dispunha de uma relação de 36 medicamentos, sendo 29 princípios ativos que atendiam às patologias mais frequentes no Estado. Destinava-se a municípios com população de até 20.000 habitantes ${ }^{16}$. Marcado pela lógica do período que antecede a PNAF, a AF no estado resumia-se à aquisição e distribuição de medicamentos.

Nos anos 2000, com a edição de importantes marcos regulatórios (PNM, PNAF, Pacto pela Saúde) e importantes evidências científicas discutidas por diferentes autores ${ }^{10,17,18}$, a Secretaria Estadual de Saúde do estado de Minas Gerais insere alterações estruturais no seu organograma criando a Superintendência de Assistência Farmacêutica (SAF), organizada nas diferentes diretorias responsáveis pelos componentes da AF: medicamentos básicos, medicamentos estratégicos e medicamentos de alto custo. Criou, ainda, o Plano Estadual de Estruturação da Rede de Assistência Farmacêutica, que incluía o Programa Rede Farmácia de Minas Gerais (RFMG). Essas inovações foram fortalecidas com a edição, em 2008, da Resolução SES/MG no 1.416, que definiu os critérios de adesão pelos municípios, o padrão obrigatório das farmácias comunitárias e os valores dos incentivos financeiros para a sua construção e para contratação de profissional farmacêutico ${ }^{15}$. 
Na mesma lógica dos programas em nível nacional, o Programa Farmácia de Minas dividiu-se em três componentes: i) Medicamentos para Atenção Primária à Saúde; ii) Medicamentos de Alto Custo; e iii) Medicamentos Estratégicos, estabelecendo, como prioridade, os municípios com população inferior a 10.000 habitantes $^{19}$. Em 2009, o programa passou a abranger os municípios com até 30.000 habitantes ${ }^{20}$.

Nessa perspectiva, a Secretaria de Estado de Saúde de Minas Gerais (SES/MG) propôs intervenções nas etapas do ciclo de Assistência Farmacêutica objetivando a otimização do acesso aos medicamentos. No tocante à programação destes, esta secretaria implantou o Sistema Integrado de Gerenciamento da Assistência Farmacêutica (SIGAF) nas Gerências Regionais de Saúde (GRS), nas farmácias de medicamentos excepcionais e nas farmácias comunitárias dos municípios.

Também, no ano de 2008, a Deliberação da Comissão Intergestores Bipartite (ClB) SUS/MG no 415 pactuou, no âmbito do estado, o financiamento do Componente Básico da Assistência Farmacêutica e atualizou a Relação Estadual de Medicamentos (RESME) para a Atenção Primária à Saúde e dos Insumos para os programas de Diabetes Mellitus e Saúde da Mulher, estabelecendo as contrapartidas do estado e dos municípios ${ }^{21}$.

Em 2013, a deliberação da CIB-SUS/MG no 1.610 aprovou o financiamento do Componente Básico do Bloco da Assistência Farmacêutica (CBAF), composto por medicamentos e insumos a serem utilizados na atenção primária em saúde ${ }^{22}$.

A reestruturação da Assistência Farmacêutica em Minas Gerais possibilitou ainda a ampliação e atualização da RESME, baseada na Relação Nacional de Medicamentos Essenciais (RENAME) e no perfil epidemiológico desse estado. No entanto, a amplitude territorial e as diferenças loco regionais, falta de formalização da AF nas esferas subnacionais e a incorporação acrítica de medicamentos na Lista de Medicamentos Essenciais (LME) são fatores que fragilizam e distorcem o conceito de medicamentos essenciais, assim como a adesão dos prescritores à Relação Municipal de Medicamentos (REMUME) ${ }^{23}$. O Relatório Final de Auditoria Operacional de Assistência Farmacêutica - Programa Farmácia de Minas (2013) também evidencia e reforça tais fragilidades, acrescidas da falta de atuação dos conselhos estadual e municipais de saúde na formulação da política de AF e a deficiência no controle da execução dos serviços de atenção básica, em especial da Assistência Farmacêutica.

Na tentativa de rever a lógica ainda presente da gestão da AF em Minas Gerais centrada na aquisição e distribuição de medicamentos, foi criado, em 2016, o Programa Farmácia de Todos. Esse programa objetiva a qualificação dos serviços farmacêuticos vinculados à rede de farmácias públicas, por meio do Programa do Cuidado Farmacêutico; a proposta do abastecimento regular de medicamentos essenciais aos serviços de saúde, pela regionalização da assistência farmacêutica; a estruturação adequada para atendimento humanizado e armazenamento dos medicamentos; e o fortalecimento do Sistema de Informação em Assistência Farmacêutica (SIGAF) ${ }^{24}$. 
Cabe destacar que esse programa propõe a inserção do profissional farmacêutico na Atenção Primária à Saúde (APS), com vistas à organização das redes de cuidado.

Assim, tendo como pano de fundo a AF no Brasil e em Minas Gerais, a proposta do presente estudo foi analisar a experiência da assistência farmacêutica no município de Juiz de Fora, município-polo da macrorregião sudeste de Minas Gerais.

\section{METODOLOGIA}

Esta pesquisa parte de uma revisão bibliográfica e avança pelo campo da investigação documental. Do ponto de vista metodológico, o estudo se fundamenta na análise de documentos oficiais (leis, regulamentos, entre outros), pessoais (cartas diários, autobiografias) e públicos (livros, jornais, revistas e discursos) como fonte de informações.

Foram utilizados os Planos Municipais de Saúde (PMS) de Juiz de Fora/MG dos seguintes períodos: 1988; 1997; 2002/2005; 2006/2009; 2010/2013; e 2014/2017. A obtenção dos dados referentes aos anos de 1988 e 1997 foi realizada in loco no Conselho Municipal de Saúde (CMS) de Juiz de Fora, por meio de arquivo digital, e os dados das décadas de 2000 foram obtidos por meio de arquivo eletrônico do CMS.

Ao tomar como base os PMS, foram identificados os dados relacionados ao estudo em questão e, posteriormente, feita a classificação dentro do tema características da Assistência Farmacêutica (AF), a fim de possibilitar a interpretação e análises desses dados.

Para essa categorização foram utilizados os indicadores estabelecidos pela Organização Pan-Americana da Saúde (OPAS), para a avaliação da AF no Brasil: indicador de acesso, de qualidade e de uso racional de medicamentos ${ }^{25}$.

Apesar de existirem outros mecanismos de controle e participação social, optouse por trabalhar apenas com os PMS, o que traz ineditismo e relevância ao presente estudo. Essa opção metodológica refere-se ao fato de que essa fonte primária expressa as decisões tomadas no âmbito do controle social, em relação à AF no município de Juiz de Fora.

Assim, os PMS possibilitaram um novo olhar para área da saúde e de medicamentos, tendo como cenário as relações estabelecidas entre o Sistema Único de Saúde, a Política Nacional de Medicamentos e a Política Nacional de Assistência Farmacêutica.

\section{RESULTADOS}

As principais características dos Planos Municipais de Saúde, editados no período de 1988 a 2014, sendo que este último com vigência para o quadriênio 2014-2017, estão descritas no Quadro 1 abaixo, utilizando descritores definidos pela OPAS. 
Quadro 1 - Principais características da AF nos Planos Municipais de Saúde de Juiz de Fora - período 1988 a 2017

\begin{tabular}{|c|c|c|}
\hline PERÍODO & Temas relacionados aos indicadores da AF & Atores envolvidos \\
\hline 1988 & $\begin{array}{c}\text { Dispensação de medicamentos; Pesquisa e produção de } \\
\text { insumos; Vigilância Sanitária }\end{array}$ & $\begin{array}{l}\text { Equipe da Secretaria } \\
\text { Municipal de Saúde }\end{array}$ \\
\hline 1997 & $\begin{array}{l}\text { - Distribuição de medicamentos; Vigilância Sanitária; } \\
\text { Comissão de Farmácia e Terapêutica; } \\
\text { - Desenvolvimento, com conselhos locais, atividades sobre } \\
\text { uso e abusos dos medicamentos. }\end{array}$ & $\begin{array}{l}\text { Equipe da Secretaria } \\
\text { Municipal de Saúde } \\
\text { (SMS), com inserção } \\
\text { de um representante } \\
\text { da comunidade }\end{array}$ \\
\hline $2002 / 2005$ & $\begin{array}{l}\text { - Organização da AF; Insuficiência de farmacêuticos } \\
\text { capacitados para gerir a AF; } \\
\text { - Interrupção no fornecimento de medicamentos; Baixa } \\
\text { resolutividade no sistema; Integração entre a AF, Vigilância } \\
\text { Sanitária e demais programas; } \\
\text { - Comissão Municipal de Assistência Farmacêutica (CMAF); } \\
\text { - Divulgação das informações sobre a política de } \\
\text { medicamentos junto à equipe de saúde e à comunidade. }\end{array}$ & $\begin{array}{l}\text { SMS, com participação } \\
\text { e revisão do Conselho } \\
\text { Municipal de Saúde } \\
\text { (CMS) }\end{array}$ \\
\hline $2006 / 2009$ & $\begin{array}{l}\text { - Fragmentação e desarticulação no acesso aos } \\
\text { medicamentos; Irregularidade e atrasos na entrega } \\
\text { dos medicamentos produzidos pela FUNED; Aumento } \\
\text { de mandatos judiciais para compra de medicamentos; } \\
\text { Interrupção no fornecimento, baixa resolutividade no } \\
\text { sistema; } \\
\text { - Adequação dos recursos humanos, em todos os níveis } \\
\text { de atenção; Comissão de Farmácia e Terapêutica; Criação } \\
\text { do Núcleo de Assistência Farmacêutica; Funcionário } \\
\text { específico para serviço de farmácia nas unidades de saúde; } \\
\text { - Incrementar o projeto Ensino/Serviço (FF/UFJF/PJF). }\end{array}$ & \multirow{3}{*}{$\begin{array}{l}\text { Comissão paritária } \\
\text { com membros do } \\
\text { planejamento da SMS } \\
\text { e CMS }\end{array}$} \\
\hline $2010 / 2013$ & $\begin{array}{l}\text { - Presença do DAF; } \\
\text { - Insuficiência de profissionais farmacêuticos; } \\
\text { - Dispensação feita por profissionais não qualificados; } \\
\text { - Condições físicas precárias das farmácias; } \\
\text { - Falta de programação e recursos financeiros; } \\
\text { - Rever e divulgar a REMUME; } \\
\text { - Incentivar e adotar protocolos e diretrizes terapêuticas; } \\
\text { - Elaborar pareceres técnicos para solicitação de } \\
\text { medicamentos por meio de mandados judiciais; } \\
\text { - Definir critérios para o uso racional de medicamentos. }\end{array}$ & \\
\hline $2014 / 2017$ & $\begin{array}{l}\text { - Gestão de Abastecimento de Medicamentos e Insumos } \\
\text { nas UAPS; } \\
\text { - Responsabilizar o almoxarifado pelo armazenamento; } \\
\text { - Diminuir perda de medicamentos e insumos; } \\
\text { - Contratar profissional para AF nas UAPS; } \\
\text { - Eliminar o estoque nas UAPS (mínimo 20\% dos itens } \\
\text { prioritários) para o atendimento; } \\
\text { - Suporte técnico, supervisão sistemática, treinamentos do } \\
\text { DAF à AP/UAPS. }\end{array}$ & \\
\hline
\end{tabular}

Fonte: elaborado pelas autoras 
O Plano Municipal de Saúde (PMS) de 1988 tratou da AF no tocante à dispensação de medicamentos e à pesquisa e produção de insumos, enquanto o PMS de 1997 discorreu sobre a distribuição e dispensação de medicamentos e da inserção de uma Comissão de Farmácia e Terapêutica.

O PMS 2002/2005 incorporou as discussões do Plano Municipal de Assistência Farmacêutica, discutido isoladamente em 1999. Esse plano tinha como propostas: a) integração interinstitucional: os diversos atores presentes no cenário da Assistência Farmacêutica (produção, comercialização e dispensação e usuários de medicamentos) como base para a abordagem de uma Política Municipal de Assistência Farmacêutica (PMAF); b) mobilização dos profissionais da área da saúde e da comunidade para educação e difusão dos princípios que norteiam o uso racional de medicamentos; c) parceria com universidades e instituições formadoras de recursos humanos para educação continuada dos profissionais envolvidos na AF; d) articulação com a SES/MG; e) elaboração da PMAF em conformidade com o relatório final da I Conferência Municipal de Assistência Farmacêutica, realizada em $2002^{26}$.

Suas metas propunham a continuidade das ações da Comissão Municipal de Assistência Farmacêutica (CMAF); capacitação dos profissionais farmacêuticos e de nível médio; definição de parâmetros necessários à programação e elaboração de um manual sobre o uso racional de medicamentos e correlatos, incluindo revisão do guia farmacoterapêutico do SUS-JF; aquisição de $100 \%$ dos insumos essenciais básicos constantes na padronização de medicamentos elaborados pela CMAF; reposição de $100 \%$ das necessidades de insumos básicos segundo a programação; controle automatizado de estoque que inclua ponto de reposição, consumo médio mensal, lote de compra, com emissão automática de requisição de compra.

Já o PMS 2006/2009 tratou da fragmentação e desarticulação no acesso aos medicamentos; irregularidade e atrasos na entrega dos medicamentos produzidos pela FUNED; aumento de mandatos judiciais para compra de medicamentos; interrupção no fornecimento; baixa resolutividade no sistema; criação do Núcleo de Assistência Farmacêutica; e incremento do projeto Ensino/Serviço (parceria da Prefeitura de Juiz de Fora com a Faculdade de Farmácia da Universidade Federal de Juiz de Fora (FF/UFJF/PJF) ${ }^{27}$.

O PMS 2010/2013 discorreu sobre as ações do Departamento de Assistência Farmacêutica (DAF), implantado em 1999. Além disso, abordou a insuficiência do número de farmacêuticos nas UBS do município; a dispensação de medicamentos realizada por profissionais não qualificados; as condições físicas precárias das farmácias do município e a falta de uma programação qualificada dos medicamentos e dos recursos financeiros. Propôs, ainda, a implantação do Núcleo de Apoio à Saúde da Família (NASF), com a presença do farmacêutico; a busca de apoio para elaborar a PMAF; a revisão e divulgação da REMUME; o incentivo e a adoção de protocolos e diretrizes terapêuticas; 
a elaboração dos pareceres técnicos para a solicitação de medicamentos por meio de mandados judiciais; a definição de critérios para o uso racional, a readequação das etapas da $A F$, a reestruturação da farmácia Central, almoxarifado central e todos os pontos de dispensação de medicamentos do município; a ampliação dos recursos humanos, a gestão das farmácias pelo farmacêutico, a adoção de software para o controle de estoque; a instituição da comissão de farmacoterapêutica, a normatização da prescrição e dispensação de medicamentos, a execução da contrapartida municipal do Componente Básico para Assistência Farmacêutica (CBAF) e para os diabéticos; a execução dos recursos do CB (federal), o acompanhamento da execução das contrapartidas (federal e estadual), a garantia do acesso aos serviços da farmácia popular, a elaboração e edição do formulário terapêutico, a implantação do SIGAF e a revisão anual da programação da $\mathrm{AF}^{28}$.

A gestão de abastecimento de medicamentos e insumos foi proposta pelo PMS 2014/2017. Esse plano propõe garantir agilidade no abastecimento de medicamentos e insumos nas Unidades Básicas de Saúde (UBS); estabelecer comunicação eficaz com as UBS; criar planilhas para o controle do estoque, normas e rotinas de pedidos, promovendo a rotatividade de medicamentos e insumos, evitando desperdício; reserva técnica de medicamentos e insumos; manter histórico dos pedidos atualizados fornecendo dados para subsidiar tomadas de decisões no ajuste do quantitativo a ser enviado às UBS; eliminar estoques nas UBS, mantendo estoque de $20 \%$ dos itens prioritários para o atendimento, responsabilizando o almoxarifado pela tarefa de armazenamento de medicamentos e insumos; estabelecer parceria com o DAF para que este responda tecnicamente pela gestão de medicamentos e insumos (normatizações, controle, dispensação, armazenamento e outros); contratar profissional técnico em farmácia para as UBS; e solicitar suporte técnico, supervisão sistemática e treinamentos do DAF à APS/UBS ${ }^{29}$.

\section{DISCUSSÃO}

O primeiro registro de atividades de $\mathrm{AF}$, nos documentos formais da SMS de Juiz de Fora, data da edição do primeiro PMS ainda no final da década de 1980. Neste período a discussão sobre atividades de promoção à saúde estava fortemente influenciada pelas discussões da 8 a Conferência Nacional de Saúde, bem como das discussões da Assembleia Nacional Constituinte e da inserção do capitulo da saúde na Constituição Federal de $1988^{30}$.

Nas décadas seguintes, com a crescente institucionalização da AF, ações políticonormativas tiveram repercussão no financiamento, estruturação e organização dos serviços farmacêuticos nos diversos espaços operativos do SUS.

$A$ análise dos documentos aponta avanços em alguns marcos regulatórios na $\mathrm{AF}$ em Juiz de Fora, principalmente no final da década de 1990 e nos anos iniciais de 2000. Merece destaque o Plano Municipal de Assistência Farmacêutica (1999), com efetiva 
participação social, garantindo avanços no processo de institucionalização da AF nas discussões do CMS e a criação municipal do Departamento de Assistência Farmacêutica (DAF), pelo Decreto 9.747/09¹. Na formulação de suas políticas, o DAF instituiu as seguintes portarias municipais: Portaria 1.443/09, que insere a Comissão de Farmácia e Terapêutica (CFT) para atuar no âmbito das unidades próprias, contratadas e conveniadas com o SUS; Portaria 1.444/09, que nomeou a Comissão Municipal de Assistência Farmacêutica para elaboração da Política Municipal de Assistência Farmacêutica de Juiz de Fora; a Portaria 1.445/09, que normatizou a prescrição e a dispensação de medicamentos no âmbito das unidades próprias, contratadas e conveniadas com o SUS, no município de Juiz de Fora; e ainda, em 2009, o Conselho Municipal de Saúde (CMS) aprovou a nova REMUME.

Na prática, essas iniciativas pouco interferiram na efetividade da AF no município. Considerando a complexidade da AF como uma estratégia da integralidade da atenção no sistema de saúde, observa-se um desconhecimento da maioria dos gestores pela área. No caso da avaliação dos PMS, construídos pela articulação da gestão com o controle social, observa-se ainda que, apesar da sensibilidade dos conselheiros no que se refere às políticas públicas de saúde, ocorre um desconhecimento das principais recomendações e portarias que regulamentam os componentes da AF nos diferentes níveis de atenção à saúde.

Estudos relacionados ${ }^{31,32}$ destacam o papel do controle social na definição dos critérios e forma de acesso da população aos medicamentos. Assim, seria relevante identificar a definição pelo gestor e a pactuação no Conselho de Saúde, não só da Relação de Medicamentos a ser ofertada aos usuários, como dos demais instrumentos previstos no Decreto no 7.508/11, para efetivação da AF no município ${ }^{12}$.

No ano de 2013, a Portaria GM/MS no 2.135/13 atualiza as normativas para elaboração do PMS, a Programação Anual de Saúde e o Relatório Anual de Gestão 33 . Além disso, os instrumentos da gestão pública também precisam contemplar a AF na Programação Plurianual, Leis Orçamentárias e Relatórios de Execução e Gestão Fiscal.

Ainda que a organização da AF no município tenha evoluído no período, as atividades previstas de $\mathrm{AF}$ não foram cumpridas e os relatórios de gestão, quando elaborados, não tratam dessas questões. Ademais, as discussões e encaminhamentos estão centrados apenas no binômio aquisição/distribuição de medicamentos.

No que se refere à estruturação da AF nos diferentes pontos da rede de atenção municipal, observa-se a discussão e encaminhamentos do CMS sobre a implantação do Núcleo de Apoio à Saúde da Família (NASF), previsto no PMS 2010/2013, destacando a inserção do farmacêutico. O gestor municipal, contrariando os anseios da população e as evidências da literatura, optou pela não implantação deste equipamento na rede municipal de saúde. Destaca-se que os NASF foram criados pela Portaria GM/MS no 154/08, para apoiar a Estratégia de Saúde da Família (ESF), ampliando sua abrangência, resolutividade, territorialização e regionalização das ações da APS $^{34}$. 
A criação desse núcleo poderia trazer melhoria na AF prestada no município, com ênfase na integralidade das ações. Como afirmam Oliveira e Leite ${ }^{35}$, os NASF, ao serem implantados, abriram novas perspectivas para a $A F$, em relação ao trabalho multiprofissional e à equipe de atenção primária, qualificando a dispensação de medicamentos e o cuidado farmacêutico.

Outros desafios que devem ser enfrentados dizem respeito à falta, ao estoque muitas vezes inadequado e ao desperdício de medicamentos e de outros insumos nas UBS, o que pode comprometer toda a logística da AF e a resolutividade das ações de saúde. Em 2003, Alves ${ }^{36}$ já apontava tal situação, bem como a ausência do farmacêutico nas unidades de saúde de Juiz de Fora e, corroborando seus achados, em 2015, o relatório da Comissão de Saúde da Câmara de Vereadores de Juiz de Fora indicou uma falta constante de medicamentos nas unidades de saúde para a assistência farmacêutica básica.

Pari passu com os indicadores de estrutura da OMS, alguns avanços na AF municipal merecem destaque: revisão e difusão da REMUME perante aos prescritores, usuários, Conselho Municipal de Saúde, Ministério Público e Poder Judiciário; institucionalização e efetivação da Comissão Municipal de Farmácia e Terapêutica; acompanhamento e qualificação do Setor de Mandados Judiciais da SMS, com vistas a demandas advindas da judicialização da saúde; concurso público para farmacêuticos, qualificando a AF do SUS municipal.

Os medicamentos componentes da AF do SUS estão presentes de modo significativo nas demandas judiciais, evidenciando a existência de problemas gerenciais, como: falta de medicamentos; barreiras ao acesso; demora na avaliação das solicitações administrativas ou desatualização dos Protocolos Clínicos e Diretrizes Terapêuticas, com predomínio de medicamentos que não fazem parte das listas oficiais ${ }^{37}$. Também, segundo Pepe et al. ${ }^{38}$, a demanda judicial por medicamentos revela, em grande parte, as deficiências e os obstáculos que devem ser superados, principalmente pelas instâncias do SUS e, em particular, na gestão da AF.

Os PMS analisados identificam a necessidade da contração de profissionais, o que só ocorreu a partir de 2016, após 14 anos das primeiras discussões no tocante ao profissional farmacêutico. Assim, até o início de 2018, foram preenchidos 30 postos de profissionais farmacêuticos, destacando a inserção deste profissional observada nas Unidades Básicas de Saúde (UBS), conforme o preconizado pelo PMS 2014/2017.

$O$ desafio de estruturação da AF ainda permanece nas definições do PMS 2018/2021. É necessário avançar no entendimento da diversidade da AF no que compete às concepções centradas tanto no controle logístico dos medicamentos, orientação ou informação sobre o uso racional, como na integralidade do cuidado farmacêutico que transcendem os arranjos legais, logísticos e técnicos na organização da assistência farmacêutica ${ }^{38-40}$. 
O envelhecimento da população brasileira e o surgimento das condições crônicas agravam a situação do município em função do aumento dos gastos com medicamentos e da sobrecarga dos outros níveis de atenção ocasionados, muitas vezes, pelo uso irracional dos medicamentos. Além disso, a crescente judicialização da saúde impacta de modo sensível o financiamento da saúde ${ }^{37-39}$.

Estudos realizados em Juiz de Fora, durante a segunda década de 2000, demonstraram que $62,5 \%$ das prescrições, respaldadas por ação judicial, eram provenientes de estabelecimentos públicos de saúde, bem como representaram 99,7\% do custo estimado de medicamentos que não eram competência de o município fornecer ou dispensar ${ }^{39,40}$. Além disso, a adesão ao tratamento medicamentoso em idosos, por meio do cuidado farmacêutico, pode resultar na melhoria da qualidade de vida desta população e na redução de custos na atenção secundária e terciária.

\section{CONSIDERAÇÕES FINAIS}

A análise dos PMS de Juiz de Fora revela que os indicadores de acesso e uso racional dos medicamentos, propostos pela OPAS ${ }^{25}$, foram inseridos na estrutura municipal. Todavia, o indicador qualidade dos medicamentos ainda se encontra incipiente.

Conclui-se que a AF no município apresenta fragilidades e potencialidades. Em relação às potencialidades, percebe-se que houve a introdução da AF no organograma da Secretaria Municipal de Saúde, por meio da criação do DAF, a implantação da CFT, a presença do farmacêutico como gestor da $A F$, o aumento do número desses profissionais, a efetivação da CFT acompanhando os processos de aquisição de medicamentos, bem como os da judicialização da saúde.

No tocante às fragilidades, observa-se, no período analisado, além da ausência do profissional farmacêutico, que só a partir de 2014 houve a contratação de uma empresa terceirizada para realizar a logística dos medicamentos no tocante ao armazenamento e distribuição de medicamentos. Isso demonstra fragilidades na gestão logística do medicamento comprometendo a AF no município, visto que tal prática fortalece o binômio aquisição/distribuição, gerando uso irracional desses insumos.

Outra fragilidade diz respeito aos sistemas de informações sobre os medicamentos e gestão da AF ainda não implantados no município de Juiz de Fora. $O$ Sistema Integrado de Gerenciamento da Assistência Farmacêutica (SIGAF) da Secretaria de Estado de Saúde de Minas Gerais ainda é incipiente, sendo utilizado somente para a gestão da AF estadual.

Além disso, a partir de 2014, a SMS começou a utilizar a parametrização nas listas de medicamentos provenientes das UBS, por meio do sistema de gerenciamento de medicamentos disponibilizado por empresa terceirizada, gerando duplicidade de informações e, consequentemente, desperdício de medicamentos. 
Assim, a integração entre a SMS e o controle social representado pelos conselhos de saúde se faz necessária. Esta integração permite avanços nos desafios que se colocam para a melhoria da AF no município visto que esses insumos são responsáveis pela melhoria das condições de saúde da população, promovendo a resolutividade das ações e serviços de saúde. Uma AF bem estruturada constitui um dos pilares para os sistemas de apoio das redes de atenção à saúde, garantindo o acesso e o uso racional de medicamentos, enquanto direito de cidadania.

\section{REFERÊNCIAS}

1. Juiz de Fora. Prefeitura Municipal de Juiz de Fora. Decreto do Executivo no 9.747, de 01/01/2009. Disponível em https://jflegis.pjf.mg.gov.br/c_periodo_exec.php.

2. Brasil. Lei no 8.080, de 19 de setembro de 1990. Dispõe sobre as condições para promoção, proteção e recuperação da saúde, a organização e o funcionamento dos serviços correspondentes e dá outras providências. Diário Oficial da União, 1990 set 20a, Sec. 1, p. 18055.

3. Brasil. Lei $n$ o 8.142, de 28 de dezembro de 1990. Dispõe sobre a participação da comunidade na gestão do Sistema Único de Saúde - SUS e sobre as transferências intergovernamentais de recursos financeiros na área da saúde e dá outras providências. Diário Oficial da União, 1990 dez 31b, Sec. 1, p. 25694.

4. Brasil. Ministério da Saúde. Portaria GM 3.916/98. Política Nacional de Medicamentos. Brasília: Ministério da Saúde, 2001.

5. Brasil. Ministério da Saúde. Secretaria de Políticas de Saúde. Departamento de Atenção Básica. Portaria 176/199. Incentivo à Assistência Farmacêutica Básica: o que é e como funciona, Departamento de Atenção Básica. Brasília: 2001 [acesso em 2017 dez 12]. Disponível em http://bvsms.saude.gov.br/bvs/publicacoes/incentivo_ assit_farm.pdf.

6. Kornis GEM, Braga MH, Zaire CEF. Os marcos legais das políticas de medicamentos no Brasil contemporâneo (1990-2006). Rev. Atenção Primária em Saúde. 2008; 11(1):85-99.

7. Brasil. Conselho Nacional de Saúde. Resolução n. 338, de 6 de maio de 2004. Aprova a Política Nacional de Assistência Farmacêutica. Diário Oficial da União. Brasília: Ministério da Saúde, 2004. Poder Executivo, Seção 1 n. 96, 2004 maio 20.

8. Brasil. Ministério da Saúde. Secretaria Executiva. Departamento de Apoio à Descentralização. Diretrizes Operacionais dos Pacto s pela Vida, em Defesa do SUS e de Gestão. v.1. Brasília: Ministério da Saúde, 2006.

9. Brasil. Portaria GM no 204/07 regulou a forma de financiamento e transferência de recursos para a s ações e serviços de saúde BRASIL. Ministério da Saúde. Portaria no 204, de 29 de janeiro de 2007. Regulamenta o financiamento e a transferência 
dos recursos federais para as ações e os serviços de saúde, na forma de blocos de financiamento, com o respectivo monitoramento e controle. Brasília: Ministério da Saúde, 2007b [acesso em 2015 dez]. Disponível em http://portal2.saude.gov.br/ saudelegis/leg_norma_pesq_consulta.cfm.

10. Correr CJ, Otuki MF, Orenzio S. Assistência farmacêutica integrada ao processo de cuidado em saúde: gestão clínica do medicamento. Rev Pan-Amaz Saude. 2011; 2(3):41-9.

11. Brasil. Lei 12.401, de 28 de abril de 2011. Altera a Lei 8.080, de 19 de setembro de 1990, para dispor sobre a assistência terapêutica e a incorporação de tecnologia em saúde âmbito do Sistema Único de Saúde - SUS. Disponível em http://portal.saude. gov.br/portal/arquivos/pdf/LEI_12401.pdf.

12. Brasil. Decreto no 7.508, de 28 de junho de 2011. Regulamenta a Lei no 8.080 , de 19 de setembro de 1990. Diário Oficial da União, 2011 jun 29b, Sec. 1, p. 1.

13. Secretaria de Saúde do Estado de São Paulo. Programa Dose Certa [acesso em 2017 jul 27]. Disponível em http://www.saude.sp.gov.br/ses/perfil/gestor/assistenciafarmaceutica/medicamentos-dos-componentes-da-assistencia-farmaceutica/ medicamentos-do-componente-basico-da-assistencia-farmaceutica/programa-dosecerta.

14. Machado-dos-Santos SC. Farmácia cidadã: integralidade, humanização e racionalidade na atenção ao paciente. In: Osorio-de-Castro CGS. (Org.). Assistência farmacêutica: gestão e prática para profissionais da saúde. Rio de Janeiro: Ed. FIOCRUZ, 2014.

15. Secretaria de Estado de Saúde de Minas Gerais (SES/MG). Superintendência De Assistência Farmacêutica. Rede Farmácia de Minas. Plano Estadual de Estruturação de Rede de Assistência Farmacêutica: uma estratégia para ampliar o acesso e o uso racional de medicamentos no SUS. Belo Horizonte: SES/MG, 2008.

16. Cosendey MAE, et al. Assistência farmacêutica na atenção básica de saúde: a experiência de três estados brasileiros. Cad. Saúde Pública. 2000; 16(1):171-82.

17. Marin N, Luiza VL, Osorio-de-Castro CGS, Machado-dos-Santos S (Org.). Assistência farmacêutica para gerentes municipais. Rio de Janeiro; Organização Pan-Americana da Saúde; 2003.

18. Osorio-de-Castro CGS (Org.). Assistência farmacêutica: gestão e prática para profissionais da saúde. Rio de Janeiro: Ed. FIOCRUZ, 2014.

19. Secretaria de Estado de Saúde de Minas Gerais (SES/MG). Resolução SES/MG n. 1.416 de 21 fev. 2008. Diário Oficial do Estado de Minas Gerais, Belo Horizonte, 2008.

20. Secretaria de Estado de Saúde de Minas Gerais (SES/MG). Resolução SES/MG n. 1.795, de 11 mar. 2009. Diário Oficial do Estado de Minas Gerais, Belo Horizonte, 2009. 
21. Secretaria de Estado de Saúde de Minas Gerais (SES/MG). Deliberação CIB-SUS/MG no 415, de $21 / 02 / 2008$.

22. Minas Gerais. Governo do Estado de Minas Gerais. Secretaria de Estado de Saúde de Minas Gerais (SES/MG). Deliberação CIB-SUS/MG № 1.610, de 16/10/2013.

23. Magarinos-Torres $\mathrm{R}$ et al. Medicamentos essenciais e processo de seleção em práticas de gestão da Assistência Farmacêutica em estados e municípios brasileiros. Ciênc. Saúde Coletiva. 2014; 19(9):3859-68.

24. Secretaria de Estado de Saúde de Minas Gerais (SES/MG). Assistência Farmacêutica no SUS [acesso em 2017 set]. Disponível em http://www.saude.mg.gov.br/ farmaciadetodos.

25. Organização Pan-Americana de Saúde, Ministério da Saúde. Avaliação da assistência farmacêutica no Brasil: estrutura, processo e resultados. Brasília: Ministério da Saúde, 2005.

26. Prefeitura Municipal de Juiz de Fora. Secretaria de Saúde. Plano Municipal de Saúde de Juiz de Fora - 2002-2005 [acesso em 2017 maio]. Disponível em https://www.pjf. mg.gov.br/.

27. Prefeitura Municipal de Juiz de Fora. Secretaria de Saúde. Plano Municipal de Saúde de Juiz de Fora - 2006-2009 [acesso em 2017 maio]. Disponível em https://www.pjf. mg.gov.br/.

28. Prefeitura Municipal de Juiz de Fora. Secretaria de Saúde. Plano Municipal de Saúde de Juiz de Fora - 2010-2013 [acesso em 2017 maio]. Disponível em https://www.pjf. mg.gov.br/.

29. Prefeitura Municipal de Juiz de Fora. Secretaria de Saúde. Plano Municipal de Saúde de Juiz de Fora - 2014-2017 [acesso em 2017 maio]. Disponível em https://www.pjf. mg.gov.br/.

30. Brasil. Constituição da República Federativa do Brasil. Texto promulgado em 05 de outubro de 1988. Brasília: Senado Federal, 1988.

31. Bruns SF, Luiza VL, Oliveira EA. Gestão da assistência farmacêutica em municípios do Estado da Paraíba (PB): olhando a aplicação de recursos públicos. Revista de Administração Pública, 2014; 48(3); 745-65.

32. Braga MH. O Controle Social do SUS Através dos Conselhos Municipais de Saúde: Um Olhar Sobre a Experiência de Juiz de Fora e seu Entorno [dissertação]. Instituto de Medicina Social: Universidade do Estado do Rio de Janeiro; 2003.

33. Brasil. Ministério da Saúde. Portaria no 2.135, de 25 de setembro de 2013. http://bvsms.saude.gov.br/bvs/saudelegis/gm/2013/prt2135_25_09_2013.html

34. Brasil. Diretrizes do NASF: Núcleo de Apoio a Saúde da Família. Ministério da Saúde, Secretaria de Atenção à Saúde, Departamento de Atenção Básica - Brasília: 2010. 
35. Oliveira JC, Leite SN. Operacionalizando a assistência farmacêutica no nível municipal. In: Osorio-de-Castro CGS. (Org.). Assistência farmacêutica: gestão e prática para profissionais da saúde. Rio de Janeiro: Ed. FIOCRUZ, 2014.

36. Alves TNP. Dispensação de medicamentos: aspectos da realidade em unidades básicas de saúde de Juiz de Fora, MG [dissertação]. Instituto de Medicina Social: Universidade do Estado do Rio de Janeiro; 2003.

37. Munck AKR. Perspectiva médico-sanitária das demandas judiciais de medicamentos em um município polo de Minas Gerais [dissertação]. Faculdade de Medicina: Universidade Federal de Juiz de Fora; 2014.

38. Pepe VLE, et al. A judicialização da saúde e os novos desafios da gestão da assistência farmacêutica. Ciênc. Saúde Coletiva. 2010; 15(5):2405-14.

39. Nunes RP. Judicialização no âmbito do Sistema Único de Saúde: um estudo descritivo sobre o custo das ações judiciais na saúde pública do município de Juiz de Fora [dissertação]. Faculdade de Medicina: Universidade Federal de Juiz de Fora; 2016.

40. Costa EA, et al. Concepções de Assistência Farmacêutica na Atenção Primária à Saúde, Brasil. Rev. Saúde Pública. 2017; 51(supl.2):1s-5s.

Submissão: abril de 2019.

Aprovação: julho de 2020. 